\title{
Hund's rule in open-shell states of two-electron systems: From free through confined and screened atoms, to quantum dots
}

\author{
Jacob Katriel $^{1}$, H. E. Montgomery ${ }^{2}$, Jr., A. Sarsa ${ }^{3}$, E. Buendía ${ }^{4}$, F. J. Gálvez ${ }^{4}$, K. D. Sen ${ }^{5}$ \\ ${ }^{1}$ Department of Chemistry, Technion - Israel Institute of Technology, Haifa, 32000 Israel \\ ${ }^{2}$ Chemistry Program, Centre College, Danville, Kentucky 40422, USA \\ ${ }^{3}$ Departamento de Física, Campus de Rabanales Edif. C2, \\ Universidad de Córdoba, E-14071 Córdoba, Spain \\ ${ }^{4}$ Departamento de Física Atómica, Molecular y Nuclear, Facultad de Ciencias, \\ Universidad de Granada, E-18071 Granada, Spain \\ ${ }^{5}$ School of Chemistry, University of Hyderabad, Hyderabad-500 046, India \\ jkatriel@technion.ac.il, ed.montgomery@centre.edu, fa1sarua@uco.es, \\ buendia@ugr.es, galvez@ugr.es, kds77@uohyd.ac.in
}

DOI 10.17586/2220-8054-2019-10-1-31-41

\begin{abstract}
Singly-excited singlet-triplet pairs of states of two-electron spherically symmetric systems, that are degenerate in the absence of inter-electronic repulsion, are revisited. In addition to the obvious two-electron atom we consider the two-electron quantum dot confined by either a harmonic potential or by an infinite spherical well, the confined two-electron atom, and three variants of an atom immersed in a plasma, modeled by the screened Coulomb (Debye) potential. The validity of Hund's multiplicity rule is confirmed, and the contribution of the interparticle repulsion energy to the singlet-triplet splitting is examined. One feature that all these systems share is that the triplet wave function is contracted relative to that of the corresponding singlet. This feature, which is a consequence of the virial theorem, affects both the behavior of the outer electron ionization energies and the relative magnitudes of the inter-particle repulsion energies in the singlet $v s$. the triplet. Whereas in atomic highly positive ions the interelectronic repulsion is lower in the triplet than in the corresponding singlet state, this ordering is reversed in neutral atoms. Such reversal does not take place in quantum dots. Confined and screened systems exhibit more nuanced behavior. The analysis utilizes appropriate variants of the virial and Hellmann-Feynman theorems.
\end{abstract}

Keywords: two-electron quantum dots, confined two-electron atom, screened coulomb potential, Hund's multiplicity rule, Virial theorem, Hellmann-Feynman theorem.

Received: 25 January 2019

Revised: 19 February 2019

\section{Introduction}

The earliest theoretical framework allowing an interpretation of Hund's heuristic rules [1] concerning the energetic ordering of atomic open-shell multiplets, and examination of their validity, was developed by Slater [2]. A generalization of Hund's rules that is consistent with Slater's treatment was developed by Morgan and Kutzelnigg [3, 4]. Slater's treatment assumed that the different states to which each electronic configuration gives rise can be treated in terms of a common set of one-electron orbitals. Within this scheme, the differences among the energies of these different states is entirely due to the corresponding differences in the magnitudes of the interelectronic repulsions. Hence, the virial theorem is violated, with different states having a common kinetic energy but different total energies.

Going beyond this frozen orbital approximation, an intriguing feature is observed. While the splitting of the states within a common configuration is driven by the interelectronic repulsion term in the Hamiltonian, the order of the calculated expectation values of this term is sometimes reversed relative to the order of the total energies. This reversal was reported for the first time by Davidson [5], studying singly excited states of He within the Hartree-Fock framework, in which the orbitals are optimized for each term individually. Later Hartree-Fock calculations [6] confirmed that the interelectronic repulsion energy is higher in the ${ }^{3} P$ than in the ${ }^{1} P$ state for all group II atoms $(\mathrm{Be}, \mathrm{Mg}, \mathrm{Ca}, \mathrm{Sr}$ and $\mathrm{Ra}$ ), where one of the two outermost $n s$ electrons is excited into the $n p$ orbital. Similar observations had been reported in the late sixties and early seventies with respect to other atoms as well as to open shell molecules. An interpretation emphasizing the role of the virial theorem and of the interaction between inner and outer shells was presented in [7] and reviewed in [8]. It was argued that correlation (i.e., beyond Hartree-Fock features) does not play a qualitatively significant role in this context. Moreover, the absence of an inner shell in systems such as doubly excited He in which the two electrons are specified by means of the 
same principal quantum number, was argued to exclude a reversal of the relative magnitudes of the interelectronic repulsion energy between states corresponding to common configurations of this type (such as $(2 s 2 p)^{1,3} P$ or $(2 p)^{2}{ }^{1} D^{3} P{ }^{1} S$ ). Some evidence supporting this claim was presented. Progress in the treatment of doubly excited states within the He isoelectronic sequence allowed, many years later, a study that suggested that correlation does play a significant qualitative role, yielding a reversal in the magnitudes of the interelectronic repulsion energies towards the low end of the isoelectronic sequence [9], despite the claim mentioned above. A comprehensive study of multiplets of neutral atoms within Hartree-Fock theory was undertaken by Koga and Koshida [10], establishing the rather universal validity of the reversal of the high- $v s$. low-multiplicity interelectronic repulsion in neutral atoms. Although positively charged atomic ions have not been studied nearly as extensively, we can conclude on sound theoretical grounds that the ordering of interelectronic repulsion energies must reverse into the "naively" expected one (the interelectronic repulsion is smaller in the lower energy state) upon increase of the nuclear charge, along isoelectronic sequences.

Application of the virial theorem suggested, rather early on, that reversal of the relative magnitudes of the interelectronic repulsion energies in different multiplicity states belonging to a common configuration is possible [7]. The proof that this reversal is inevitable was only proposed many years later [11].

During the last decade or two, growing interest in electrons "trapped" within nanoscale cavities in solids, referred to as "quantum dots", has drawn attention to the spectroscopic properties of such systems. Evidence has been accumulating that these systems typically satisfy Hund's rule, higher spin states tending to be lower in energy than corresponding lower spin states within a common configuration. However, unlike atoms, evidence has been presented indicating that these systems do not exhibit a reversal of the magnitudes of the interelectronic repulsion energies. Indeed, such reversal does not take place in the two-electron harmonically confined quantum dot [12,13], a fact that could also be accounted for analytically [11].

The two-electron atom in a spherical cavity was recently explored, interpolating between the free atom and a pair of electrons in an infinite spherical well [14]. Atomic-like behavior (reversal of the singlet vs. triplet interelectronic repulsion energies) was observed at large cavity radii, where the Coulomb nuclear attraction regulates the extension of the wave function, whereas no such reversal takes place in smaller cavities.

This issue was also explored for open-shell screened atoms, in which the Coulomb potentials were replaced by Debye (Yukawa) type potentials [15]. Three variants of the two-electron Debye atom were considered:

1. The fully-screened (FS) two-electron Debye atom, whose Hamiltonian is:

$$
\mathcal{H}_{D}=-\frac{1}{2}\left(\nabla_{1}^{2}+\nabla_{2}^{2}\right)-Z\left(\frac{\exp \left(-\lambda r_{1}\right)}{r_{1}}+\frac{\exp \left(-\lambda r_{2}\right)}{r_{2}}\right)+\frac{\exp \left(-\lambda r_{12}\right)}{r_{12}},
$$

where $\lambda$ is the screening constant.

2. The screened nuclear attraction (SNA) Hamiltonian:

$$
\mathcal{H}_{d}=-\frac{1}{2}\left(\nabla_{1}^{2}+\nabla_{2}^{2}\right)-Z\left(\frac{\exp \left(-\lambda r_{1}\right)}{r_{1}}+\frac{\exp \left(-\lambda r_{2}\right)}{r_{2}}\right)+\frac{1}{r_{12}},
$$

3. The screened interelectronic repulsion (SIR) Hamiltonian:

$$
\mathcal{H}_{\text {int }}=-\frac{1}{2}\left(\nabla_{1}^{2}+\nabla_{2}^{2}\right)-Z\left(\frac{1}{r_{1}}+\frac{1}{r_{2}}\right)+\frac{\exp \left(-\lambda r_{12}\right)}{r_{12}} .
$$

The aim of the present article is to provide a synthetic overview of all these studies. We start by presenting rigorous relations between the interelectronic repulsion energy and derivatives of simple functions of the total energy, depending linearly on the latter. These relations follow by application of appropriate versions of the virial and the Hellmann-Feynman theorems. The linear dependence mentioned above means that the same relations hold for the energy differences between different states of the pertinent system. Homogeneous potentials are dealt with in Section 2, allowing the treatment of the free Coulomb atom and the harmonic quantum well as well as the infinite spherical well. Inhomogeneous potentials are treated in Section 3. These include the confined two-electron atom as well as the systems that involve Debye screening of the nuclear attraction, the interelectronic repulsion, or both. These relations are of particular interest for pairs of states for which the corresponding function of the energy difference is non-monotonic, yielding a vanishing first derivative (i.e., reversal in the sign of the difference of interelectronic repulsion energies) somewhere along the isoelectronic sequence. By considering the asymptotic behavior of the energy differences at both the high and low ends of the isoelectronic sequence, we show that such non-monotonicities generically arise within open-shell atomic isoelectronic sequences. On the other hand, confined few-electron systems ("quantum dots"), that remain bound in the weak attractive potential limit, do not give rise to such non-monotonicities. This provides a "kinematic" interpretation of the sign reversal of differences 
of interelectronic repulsion energies, as opposed to the "dynamical" interpretations (right or wrong) that have been proposed. This analysis leaves open the possibility of reversal of interelectronic repulsion energies in the weak attractive potential limit of non-confining quantum dots, such as the Gaussian or finite spherical well, that exhibit analogues of a lower critical charge at which the system ceases to be bound.

Computational results for the various systems specified above are reviewed in Sections 4-8, confirming the analysis briefly reviewed above. For details of the computational procedures used we refer to the original publications.

Finally, we emphasize that the formal relations presented in sections 2 and 3 have a much broader relevance than the applications actually considered; the analysis of the He isoelectronic sequence is an illustration that can be extended to any open-shell, many-electron atomic isoelectronic sequence. Hence, our analysis establishes that the reversal of the relative magnitudes of the interelectronic repulsion energies in atomic multiplets corresponding to a common configuration is inevitable, while the absence of such reversal in many-electron quantum dots with confining potentials is equally universal.

\section{The virial and Hellmann-Feynman theorems for homogeneous potentials}

We consider the two-electron Hamiltonian:

$$
\mathcal{H}=-\frac{1}{2}\left(\nabla_{1}^{2}+\nabla_{2}^{2}\right)+\frac{1}{r_{12}}+\frac{1}{\nu}\left(\left(\frac{r_{1}}{R}\right)^{\nu}+\left(\frac{r_{2}}{R}\right)^{\nu}\right),
$$

noting that the generalization to $N$ electrons is straightforward. For an eigenstate $\Psi$ the energy components are:

The kinetic energy

$$
\begin{array}{ll}
\text { The kinetic energy } & T=-\frac{1}{2}\left\langle\Psi\left|\left(\nabla_{1}^{2}+\nabla_{2}^{2}\right)\right| \Psi\right\rangle, \\
\text { The one-body potential energy } & H=\frac{h}{\nu}, \quad \text { where } h=\left\langle\Psi\left|\left(\frac{r_{1}}{R}\right)^{\nu}+\left(\frac{r_{2}}{R}\right)^{\nu}\right| \Psi\right\rangle,
\end{array}
$$$$
\text { The interelectronic repulsion energy } C=\left\langle\Psi\left|\frac{1}{r_{12}}\right| \Psi\right\rangle \text {. }
$$

The total energy is:

$$
E=\langle\Psi|\mathcal{H}| \Psi\rangle=T+C+H
$$

Coordinate scaling, followed by minimization of the energy with respect to the scaling parameter, yields the virial theorem in the form:

$$
2 T+C-h=0 .
$$

The Hellmann-Feynman theorem, applied to the parameter $R$, yields:

$$
h=-R \frac{d E}{d R} .
$$

Combining (5), (6) and (7) we obtain:

$$
C=2 E+\frac{\nu+2}{\nu} R \frac{d E}{d R}=\frac{\nu+2}{\nu} R^{\frac{2-\nu}{2+\nu}} \frac{d}{d R}\left(R^{\frac{2 \nu}{\nu+2}} E\right) .
$$

We assume that $\Delta E$, the difference between the energies of some pair of eigenstates of the Hamiltonian (4), is known as a function of the parameter $R$ (in practice, this amounts to knowing it for a sufficiently dense set of values of $R$ ). Then, the differences between the interelectronic repulsion energies, $\Delta C$, can be evaluated by applying (8) to $\Delta E$. For a pair of related states whose difference of energies satisfies $\Delta E>0$, if it can be shown that $\left(R^{\frac{2 \nu}{\nu+2}} \Delta E\right)$ is non-monotonic as a function of $R$ then the interelectronic repulsion difference reverses sign at the value of $R$ at which $\left(R^{\frac{2 \nu}{\nu+2}} \Delta E\right)$ is extremal.

We shall consider the following special cases:

\subsection{The Coulomb potential, $\nu=-1$}

In this case $R$ can be identified with $Z$, the nuclear charge. We obtain:

$$
\begin{aligned}
& T=-E, \\
& H=Z \frac{d E}{d Z} \text {, } \\
& \text { and } \\
& C=-Z^{3} \frac{d}{d Z}\left(\frac{E}{Z^{2}}\right) \text {. }
\end{aligned}
$$


2.2. The harmonic potential, $\nu=2$

In this case we relate $R$ to the harmonic force constant $k$ via $k=\frac{1}{R^{2}}$, and obtain

$$
\begin{aligned}
T & =3 k^{\frac{4}{3}} \frac{d}{d k}\left(\frac{E}{k^{\frac{1}{3}}}\right), \\
H= & -2 k \frac{d E}{d k}, \\
& \text { and } \\
C= & -4 k^{\frac{3}{2}} \frac{d}{d k}\left(\frac{E}{\sqrt{k}}\right) .
\end{aligned}
$$

The expression for $C$, the expectation value of the interelectronic repulsion, can be written in terms of the frequency $\omega$, related to the force constant via $k=\omega^{2}$. One obtains:

$$
C=-2 \omega^{2} \frac{d}{d \omega}\left(\frac{E}{\omega}\right)
$$

2.3. The infinite spherical well, $\nu=\infty$

Taking the limit $\nu \rightarrow \infty$ we obtain:

$$
\begin{aligned}
T & =-\frac{d}{d R}(R E), \\
H & =0, \\
& \text { and } \\
C & =\frac{1}{R} \frac{d}{d R}\left(R^{2} E\right) .
\end{aligned}
$$

These expressions are consistent with $T+C=E$.

\section{The virial and Hellmann-Feynman theorems for inhomogeneous potentials}

\subsection{Two-parameter Hellmann-Feynman systems}

Consider the Hamiltonian:

$$
\mathcal{H}=-\frac{1}{2}\left(\nabla_{1}^{2}+\nabla_{2}^{2}\right)+\frac{1}{r_{12}}+\frac{1}{\nu_{a}}\left(\left(\frac{r_{1}}{R_{a}}\right)^{\nu_{a}}+\left(\frac{r_{2}}{R_{a}}\right)^{\nu_{a}}\right)+\frac{1}{\nu_{b}}\left(\left(\frac{r_{1}}{R_{b}}\right)^{\nu_{b}}+\left(\frac{r_{2}}{R_{b}}\right)^{\nu_{b}}\right) .
$$

Here, the potential is manifestly inhomogeneous.

The scaling argument yields the virial theorem in the form:

$$
2 T+C-h_{a}-h_{b}=0 .
$$

$T$ and $C$ are the kinetic energy and the interelectronic repulsion energy, respectively, and

$$
\begin{array}{ll}
h_{a}=\left\langle\Psi\left|\left(\frac{r_{1}}{R_{a}}\right)^{\nu_{a}}+\left(\frac{r_{2}}{R_{a}}\right)^{\nu_{a}}\right| \Psi\right\rangle, & H_{a}=\frac{h_{a}}{\nu_{a}}, \\
h_{b}=\left\langle\Psi\left|\left(\frac{r_{1}}{R_{b}}\right)^{\nu_{b}}+\left(\frac{r_{2}}{R_{b}}\right)^{\nu_{b}}\right| \Psi\right\rangle, & H_{b}=\frac{h_{b}}{\nu_{b}} .
\end{array}
$$

The Hellmann-Feynman theorem can be applied to each of $R_{a}, R_{b}$ individually, yielding:

$$
\begin{aligned}
h_{a} & =-R_{a} \frac{\partial E}{\partial R_{a}}, \\
h_{b} & =-R_{b} \frac{\partial E}{\partial R_{b}} .
\end{aligned}
$$

Using these relations we obtain:

$$
C=2 E+\frac{\nu_{a}+2}{\nu_{a}} R_{a} \frac{\partial E}{\partial R_{a}}+\frac{\nu_{b}+2}{\nu_{b}} R_{b} \frac{\partial E}{\partial R_{b}}
$$




\subsection{The confined two-electron atom}

For particles in a Coulomb potential confined by an infinite spherical well of radius $R$ [16] (and references therein) we have $\nu_{a}=-1, R_{a}=Z$ and $\nu_{b} \rightarrow \infty, R_{b}=R$. We obtain,

$$
C=2 E-Z \frac{\partial E}{\partial Z}+R \frac{\partial E}{\partial R}
$$

or

$$
C=-Z^{1-\mu_{Z}} \frac{\partial}{\partial Z}\left(Z^{\mu_{Z}} E\right)+R^{1-\mu_{R}} \frac{\partial}{\partial R}\left(R^{\mu_{R}} E\right)
$$

where $\mu_{R}-\mu_{Z}=2$.

Choosing $\mu_{Z}=0$ we obtain:

and choosing $\mu_{R}=0$,

$$
C=-Z \frac{\partial E}{\partial Z}+\frac{1}{R} \frac{\partial}{\partial R}\left(R^{2} E\right)
$$

$$
C=-Z^{3} \frac{\partial}{\partial Z}\left(\frac{E}{Z^{2}}\right)+R \frac{\partial E}{\partial R} .
$$

\subsection{The one-electron Debye-screened atom}

The one-electron Debye Hamiltonian is:

$$
\mathfrak{h}_{d}=-\frac{1}{2} \nabla^{2}-Z \frac{\exp (-\lambda r)}{r} .
$$

Let $\psi$ be an eigenfunction with eigenvalue $e$. Let:

$$
\begin{aligned}
t & =\left\langle-\frac{1}{2} \nabla^{2}\right\rangle, \\
v & =-Z\left\langle\frac{\exp (-\lambda r)}{r}\right\rangle, \\
e & =t+v, \\
u & =\lambda Z\langle\exp (-\lambda r)\rangle,
\end{aligned}
$$

where $\langle\hat{O}\rangle \equiv\langle\psi|\hat{O}| \psi\rangle$. The virial theorem:

$$
2 t+v=2 e-v=u,
$$

yields:

$$
\begin{aligned}
t & =-e+u, \\
v & =2 e-u .
\end{aligned}
$$

The Hellmann-Feynman theorem with respect to $Z$ yields

$$
Z \frac{\partial e}{\partial Z}=v
$$

and the Hellmann-Feynman theorem with respect to $\lambda$ yields

$$
\frac{\partial e}{\partial \lambda}=\frac{u}{\lambda}
$$

Using equations (18) and (19) we obtain:

$$
2 e-\lambda \frac{\partial e}{\partial \lambda}-Z \frac{\partial e}{\partial Z}=0
$$

hence,

$$
v=Z \frac{\partial e}{\partial Z}=2 e-\lambda \frac{\partial e}{\partial \lambda}=-\lambda^{3} \frac{\partial}{\partial \lambda}\left(\frac{e}{\lambda^{2}}\right)
$$

and

$$
t=-e+\lambda \frac{\partial e}{\partial \lambda}=\lambda^{2} \frac{\partial}{\partial \lambda}\left(\frac{e}{\lambda}\right)
$$




\subsection{The two-electron Debye screened atom}

3.4.1. Screened nuclear attraction Hamiltonian, $\mathcal{H}_{d}$. Let $\Psi$ be an eigenfunction of the two-electron Hamiltonian, equation (2).

$E$ is the corresponding eigenvalue. Let:

$$
\begin{aligned}
T & =\left\langle-\frac{1}{2}\left(\nabla_{1}^{2}+\nabla_{2}^{2}\right)\right\rangle, \\
L & =-Z\left\langle\frac{\exp \left(-\lambda r_{1}\right)}{r_{1}}+\frac{\exp \left(-\lambda r_{2}\right)}{r_{2}}\right\rangle, \\
C & =\left\langle\frac{1}{r_{12}}\right\rangle, \\
V & =L+C, \\
E & =T+L+C, \\
U & =\lambda Z\left\langle\exp \left(-\lambda r_{1}\right)+\exp \left(-\lambda r_{2}\right)\right\rangle .
\end{aligned}
$$

The virial theorem [17-19] yields:

$$
\begin{aligned}
T & =-E+U, \\
V & =2 E-U,
\end{aligned}
$$

the $Z$-Hellmann-Feynman theorem is

$$
\frac{\partial E}{\partial Z}=\frac{L}{Z}
$$

and the $\lambda$-Hellmann-Feynman theorem is:

$$
\frac{\partial E}{\partial \lambda}=\frac{U}{\lambda}
$$

It follows that the two components of the potential energy are given by:

$$
\begin{aligned}
C & =2 E-\lambda \frac{\partial E}{\partial \lambda}-Z \frac{\partial E}{\partial Z} \\
L & =Z \frac{\partial E}{\partial Z}
\end{aligned}
$$

Subtracting (20) from (24) we obtain:

$$
C=-2 \epsilon+\lambda \frac{\partial \epsilon}{\partial \lambda}+Z \frac{\partial \epsilon}{\partial Z}
$$

where $\epsilon=e-E$ is the first ionization energy of the two-electron system.

3.4.2. Fully screened Debye atom, $\mathcal{H}_{D}$. We follow the path described in the previous subsection, specifying only the expressions that require modification. The expectation value of the two-electron term becomes:

$$
C=\left\langle\frac{\exp \left(-\lambda r_{12}\right)}{r_{12}}\right\rangle \text {. }
$$

Equation (22) should be replaced by:

$$
U=\lambda\left\langle Z\left(\exp \left(-\lambda r_{1}\right)+\exp \left(-\lambda r_{2}\right)\right)-\exp \left(-\lambda r_{12}\right)\right\rangle .
$$

3.4.3. Screened interelectronic repulsion, $\mathcal{H}_{\text {int }}$. In this case:

$$
U=-\lambda\left\langle\exp \left(-\lambda r_{12}\right)\right\rangle
$$




\section{The two-electron atom}

\subsection{The $(1 s 2 p)^{1,3} P$ states of the He isoelectronic sequence}

The energies calculated by Accad, Pekeris and Schiff [20] for $Z=2,3, \cdots, 10$, along with the fact that the singlet-triplet energy difference vanishes at the critical charge $Z=1$ [21] yield,

$$
\Delta E \approx(Z-1)\left(0.0344887-\frac{0.0544423}{Z}+\frac{0.0081415}{Z^{2}}\right)
$$

This expression resembles the $\frac{1}{Z}$-perturbation expansion [22-25]

$$
\Delta E=\frac{224}{6561} Z-0.0840286+\cdots=0.034141137 Z-0.0840286+\cdots
$$

and the two leading coefficients crudely agree. Since, at large $Z, \Delta E$ grows linearly in $Z$, it follows that $\frac{\Delta E}{Z^{2}}$, which vanishes at $Z=1$ (since $\Delta E$ does), also vanishes asymptotically as $Z \rightarrow \infty$. Hence, $\frac{\Delta E}{Z^{2}}$ must obtain a maximum, which is found to be at $Z \approx 3.78$. From (9) (applied to $\Delta E$ ) it follows that $\Delta C$ is negative for $Z=2,3$, and positive but small at $Z=4$.

Substituting (25) into (9), we obtain:

$$
\Delta C \approx 0.0344887 Z-0.1778618+\frac{0.1877513}{Z}-\frac{0.0325661}{Z^{2}}
$$

The differences between the $a b$ initio values of the interelectronic repulsion energies in the ${ }^{3} P$ and the ${ }^{1} P$ states [20] are consistent with the values obtained by evaluating equation (26).

\subsection{The $(1 s 2 s)^{1,3} S$ states of the He isoelectronic sequence}

In complete analogy with the treatment of the $(1 s 2 p)^{1,3} P$ sequence in the previous section, we notice, using the energies calculated by Accad, Pekeris and Schiff [20] for $Z=2,3, \cdots, 10$, that $\frac{\Delta E}{Z^{2}}$ obtains a maximum (at $Z \approx 2.6$ ), which implies, because of (9), that $\Delta C$ is negative for $Z=2$. This is in agreement with the $\Delta C$ values obtained using the 21-order $\frac{1}{Z}$ variational perturbation theory expansion [26]. The value of $\Delta C$ at $Z=2$ agrees with the ab initio value, -0.018514 [27].

\section{The two-particle harmonic quantum dot: $(1 s 2 p)^{1,3} P$ states}

Using the relative-motion energies $\epsilon(n, \ell)$ evaluated by Prudente, Costa and Vianna [28] we note that the energies of the lowest ${ }^{1,3} P$ pair, $(0,1)_{r}(0,0)_{R}{ }^{3} P$ and $(0,0)_{r}(0,1)_{R}{ }^{1} P$, are given by:

$$
\begin{aligned}
& E\left({ }^{3} P\right)=\epsilon_{r}(0,1)(\omega)+\frac{3}{2} \omega, \\
& E\left({ }^{1} P\right)=\epsilon_{r}(0,0)(\omega)+\frac{5}{2} \omega,
\end{aligned}
$$

respectively. Fitting the numerical values we obtain:

$$
\frac{E\left({ }^{1} P\right)-E\left({ }^{3} P\right)}{\omega} \approx 1+\sqrt{\omega}\left(0.00166828-\frac{1.009793}{0.283907+\sqrt{\omega}}\right),
$$

in agreement with Hund's first rule, $E\left({ }^{1} P\right)>E\left({ }^{3} P\right)$. Obviously, $\lim _{\omega \rightarrow 0}\left(E\left({ }^{1} P\right)-E\left({ }^{3} P\right)\right)=0$, as each energy individually vanishes in this (no confinement) limit. As expected, the calculated energies are consistent with $\lim _{\omega \rightarrow \infty} \frac{E\left({ }^{1} P\right)}{E\left({ }^{3} P\right)}=1$. Using (11) we obtain the difference between the ${ }^{1} P$ and the ${ }^{3} P$ interelectronic repulsion energies. The ratio $\frac{\Delta E}{\omega}$ is clearly monotonically decreasing as a function of $\omega$. Consequently, the interelectronic repulsion energy in the singlet is higher than that in the triplet for all the values of $\omega$ considered. 


\section{The two-particle infinite spherical well: the ${ }^{1,3} S$ states}

The two-electron atom with nuclear charge $Z$, confined in a spherical well of radius $R$, interpolates between the free two-electron atom $(R \rightarrow \infty)$ and the spherical quantum $\operatorname{dot}(Z \rightarrow 0)$ [14].

A careful analysis of the nodal structure of the various two-electron wave functions suggests that the lowest ${ }^{3} S$ state, which corresponds to the configuration $(1 s 2 s)$, should be compared with the third ${ }^{1} S$ state, which corresponds to the same configuration. The lowest ${ }^{1} S$ state corresponds, in complete analogy with the He-like atoms and the two-electron Hooke's atom, to the ground configuration $1 s^{2}$. However, in the present case the following ${ }^{1} S$ state corresponds to the $(2 p)^{2}$ configuration.

Indeed, the $3{ }^{1} S$ and the $1{ }^{3} S$ states are rather close in energy (the latter being lower, in conformity with Hund's rule). The interelectronic repulsion energy is rather significantly lower in the latter than in the former state. Extrapolation yields: $\lim _{R \rightarrow 0} \frac{C\left(3^{1} S\right)-C\left(1^{3} S\right)}{E\left(3^{1} S\right)-E\left(1^{3} S\right)}=1$. Over the range of values of $R$ available, $R^{2}\left(E\left(3^{1} S\right)-\right.$ $\left.E\left(1^{3} S\right)\right)$, whose first derivative is related to $C\left(3{ }^{1} S\right)-C\left(1{ }^{3} S\right)$, is a monotonically increasing function of $R$, approaching zero in the limit $R \rightarrow 0$. This is consistent with $C\left(3{ }^{1} S\right)-C\left(1{ }^{3} S\right)>0$.

\section{The confined two-electron atom: the $(1 s 2 p){ }^{1,3} P$ states}

A principal distinction between the atomic isoelectronic sequence and the harmonic quantum dot is that in the former the singlet-triplet energy difference vanishes at some positive nuclear charge, below which the outermost electron is not bound. In view of the confining nature of the harmonic potential, the coincidence of the singlet and the triplet energies only takes place when the harmonic force constant vanishes. Since the infinite spherical well is even more remote from the Coulomb potential - more confining - than the harmonic well, it is safe to anticipate no reversal, as a function of the radius of the confining sphere, in the latter system as well.

To understand the transition between these two types of behaviour we consider confined open-shell manyelectron atoms. The multiplet structure suggested by the single configuration - frozen orbitals treatment of the systems that we actually study agrees with that specified by Hund's rules. Furthermore, we deliberately avoid systems that exhibit asymptotic degeneracies as $\frac{1}{Z} \rightarrow 0$. We follow the behavior of pairs of states that correspond to a common configuration, say, the singlet and triplet states that correspond to the $(1 s 2 p)$ configuration of the two-electron atom. In free space, the interelectronic repulsion is higher in the singlet for higher $Z$, and higher in the triplet for lower $Z$. Confining the system into a sphere of radius $R$ and setting $Z=0$ we still get a singlet-triplet pair, the triplet being lower in energy, but having a lower expectation value of the interelectronic repulsion, for all $R$. Our aim is to trace the transition between the case $R>0, Z=0$ and the case $R=\infty, Z>1$.

For $R \rightarrow \infty \Delta E$ is independent of $R$, so we obtain from (16):

$$
\Delta C=-Z^{3} \frac{\partial}{\partial Z}\left(\frac{\Delta E}{Z^{2}}\right) \text {. }
$$

Since $\frac{\Delta E}{Z^{2}}$ vanishes at $Z=Z_{c}$, the critical charge at which the outer electron ceases to be bound, as well as at $Z \rightarrow \infty$, and is positive in between, it must possess a maximum at some intermediate value $Z_{m}$. Hence, $\Delta C<0$ for $Z_{c}<Z<Z_{m}$, and $\Delta C>0$ for $Z>Z_{m}$.

For $Z=0$ we obtain from (15):

$$
\Delta C=\frac{1}{R} \cdot \frac{\partial}{\partial R}\left(R^{2} \Delta E\right) .
$$

Near-Hartree-Fock wavefunctions and energies were obtained for the singly excited pair of states $(1 s 2 p)^{1,3} P$, over a range of nuclear charges and confining radii. In all cases, the total energy is lower in the triplet than in the singlet. The most interesting aspect is that the difference between the expectation values of the interelectronic repulsion of the singlet and the corresponding triplet states. One notes that the interelectronic repulsion is higher in the singlet except at low $Z$ and high $R$.

For low $R$ the difference of the interelectronic repulsions between the singlet and the triplet is a decreasing function of $Z$. This is because when $Z$ increases for a constant $R$ the wavefunction contracts and the boundary becomes less significant. Since the singlet is more expanded, the boundary affects it more significantly, increasing the singlet-triplet difference of interelectronic repulsions. At high $R$, including the free atom $(R=\infty)$, the difference of interelectronic repulsions increases with increasing $Z$. This is a trivial consequence of the fact that the difference of interelectronic repulsions is, asymptotically, equal to $2 K[1 s, 2 p] Z$, where $K[1 s, 2 p]$ is the exchange integral. A minimum in the singlet-triplet difference of interelectronic repulsions as a function of $Z$ is 
noted at intermediate $R$. For $Z$ above this minimum the high $R$ behaviour is manifested, whereas for $Z$ below the minimum one is in the low $R$ regime. The location of the minimum shifts to lower $Z$ upon increase of $R$. Noticing this trend helps understand what would otherwise look to be a suspicious sequence of interelectronic repulsion differences at $R=5$. The difference of interelectronic repulsions is always a decreasing function of $R$, for constant $Z$. This trend is due to the fact that the confinement affects the singlet - that is more expanded more than the triplet, increasing the singlet interelectronic repulsion upon lowering $R$.

\section{The Debye-screened two-electron atom: the $(1 s 2 s)^{1,3} S$ states}

Finally, we consider the fully-screened (FS) two-electron Debye atom, (1), the screened nuclear attraction (SNR) Hamiltonian (2), and the screened interelectronic repulsion (SIR) Hamiltonian (3) [15]. We refer to the term $\frac{\exp \left(-\lambda r_{12}\right)}{r_{12}}$ either as the screened interelectronic repulsion or as the interparticle repulsion. The operator $\frac{1}{r_{12}}$ is referred to, as usual, as the interelectronic repulsion. We say "particle" to emphasize that the interaction is modified, although the properties (mass, spin, statistics) are those of an electron. The expectation values of these operators are referred to as the interparticle or interelectronic repulsion energies, respectively.

We find that the methodological standpoint taken by Lin et al. [29], allowing the effect of the Debye screening of the one- and the two-particle terms to be distinguished from one another, is helpful when dealing with the excited states as well. The fact that in $\mathcal{H}_{D}$ both the one-electron and the two-electron potential energy terms are screened leads to unexpected trends in the behavior of the triplet $v s$. the singlet interparticle repulsion energies. We expect $\mathcal{H}_{d}$ to behave more similarly to the confined two-electron atom. A complementary picture is offered by $\mathcal{H}_{\text {int }}$. Upon increasing the Debye screening the interelectronic repulsion is quenched and the system approaches two non-interacting hydrogen-like electrons in the $(1 s 2 s)$ configuration, with the $\lambda \rightarrow \infty$ asymptotic energy $-Z^{2}\left(\frac{1}{2}+\frac{1}{8}\right)$

and

$$
v=Z \frac{\partial e}{\partial Z}=2 e-\lambda \frac{\partial e}{\partial \lambda}=-\lambda^{3} \frac{\partial}{\partial \lambda}\left(\frac{e}{\lambda^{2}}\right)
$$

$$
t=-e+\lambda \frac{\partial e}{\partial \lambda}=\lambda^{2} \frac{\partial}{\partial \lambda}\left(\frac{e}{\lambda}\right) .
$$

The differences between the singlet and the triplet energies for the FS, the SNA and the SIR models have been examined as functions of $\frac{\lambda}{Z}$, for $Z=2,3,4,5$. The singlet is always higher than the corresponding triplet, the difference increasing with increasing $Z$. From the different versions of the $\lambda$-Hellmann-Feynman theorem it follows that the initial slopes of the singlet and the triplet energies, at $\frac{\lambda}{Z}=0$, are equal. Hence, $\Delta E$ starts horizontally, with a vanishing slope.

For the SNA atom both the singlet and the triplet energies are higher (lower in absolute value) than those of the FS atom. This is due to the higher interparticle repulsion in the former systems. The singlet-triplet energy differences are smaller for the SNA atoms than for the FS atoms.

For the SIR atom the singlet-triplet energy difference increases at low $\frac{\lambda}{Z}$, because the triplet energy is lowered more rapidly by the enhanced interparticle screening. This energy difference reaches a maximum (roughly at $\frac{\lambda_{m}}{Z} \approx \frac{0.65}{\sqrt{Z}}$ ), vanishing asymptotically at $\frac{\lambda}{Z} \rightarrow \infty$.

\subsection{The interparticle repulsion energies}

For $Z=2$ the interparticle repulsion energy is lower in the singlet over the whole range of values of the screening constant for both the FS atom and the SNA atom. For the SIR atom the difference between the singlet and the triplet interparticle repulsion energies rises, becoming positive above a certain screening constant. For $Z \geq 3$ the interparticle repulsion in the FS atom is lower in the triplet at low screening (up to $\lambda \approx 0.096$ at $Z=3$, $\lambda \approx 0.17$ at $Z=4$ and $\lambda \approx 0.208$ at $Z=5$ ), but higher in the triplet for higher screening. This reversal takes place at lower values of the screening constants for the SNA atom. While the SIR singlet interparticle repulsion energy is always higher than that of the triplet, they both vanish, asymptotically, at $\frac{\lambda}{Z} \rightarrow \infty$, where the screening quenches the interparticle repulsion. Like the ionization energy, the triplet interparticle repulsion energy approaches the asymptotic value considerably more rapidly than the singlet. 
Finally, Debye screening of the nuclear attraction enhances the reversal of the magnitudes of the singlet $v s$. the triplet interparticle repulsion energies by effectively lowering the nuclear charge, whereas Debye screening of the interparticle repulsion acts in the opposite direction. This behavior is due to the fact that the singlet wave function is more expanded, so that it samples the interparticle repulsion operator at higher values of $r_{12}$, at which the effect of the factor $\exp \left(-\lambda r_{12}\right)$ is more pronounced.

\section{Conclusions}

The behavior of the interelectronic repulsion energy in two-electron systems with a range of one-body potentials was reviewed. Particular attention was paid to the relative magnitudes of the interelectronic repulsion energies within pairs of states which, in the limit of vanishing interelectronic repulsion, belong to a common electronic configuration. Expressions for these differences of interelectronic repulsion energies in terms of derivatives of simple expressions involving differences of corresponding total energies were derived, using appropriate versions of the virial and Hellmann-Feynman theorems. In the atomic case the expressions to be differentiated were shown to be non-monotonic by considering their behavior for both $Z \rightarrow \infty$ and for $Z \rightarrow Z_{c}$ (where $Z_{c}$ is the critical charge below which the outermost electron is not bound). Hence, their derivatives yield an inevitable sign reversal in the difference of interelectronic repulsion energies, for some $Z$ that satisfies $Z_{c}<Z<\infty$.

In the confined systems the behavior at the high correlation limit of the isoelectronic sequences (when $k \rightarrow 0$ for Hooke's atom and when $R \rightarrow \infty$ for the infinite spherical well) implies a monotonic behavior of the energyrelated quantities that need to be differentiated to obtain the corresponding difference of interelectronic repulsion energies. Hence, no sign reversal is expected. These conclusions are confirmed computationally, for appropriate pairs of states.

Careful analysis indicates that the ordering and structure of electronic configurations in Hooke's atom, and even more so in the infinite spherical well, are sometimes very different from those in the atomic case, due to different degeneracies and configuration mixing. Such distinctions are important to keep in mind when trying to draw meaningful comparisons.

The lowest ${ }^{1,3} \mathrm{P}$ pair exhibits remarkable robustness in the following sense: this is the only pair of states in which both members are lowest within the subspaces of their respective symmetry types, being reasonably well described by the $(1 s 2 p)$ configuration. The $(1 s 2 s)^{1,3} S$ pair suffers from the fact that the singlet is not the lowest of its symmetry type, which makes a treatment in terms of a single configuration approximation somewhat delicate. The ${ }^{1,3} D$ states are even worse. They are nicely described in terms of the single configuration $(1 s 3 d)$ in the atomic case, but each member of the pair belongs to a different limiting configuration (the singlet being $(2 p)^{2}$ and the triplet $(1 s 3 d)$ ) in the infinite spherical well. Even in Hooke's atom the lowest ${ }^{1} D$ state involves a linear combination of two configurations that are mutually degenerate in the non-interacting limit. In view of this robustness of the ${ }^{1,3} \mathrm{P}$ pair, the single most pertinent recommendation for further study is that this pair of states should be examined more carefully for spherically symmetric quantum dots with a range of shapes and strengths, including, in particular, non-confining potentials such as a finite well or a Gaussian potential. This is the context in which the least ambiguous results are likely to emerge.

The lowest energy pair of singly-excited states, $(1 s 2 s)^{1,3} S$, is investigated for three types of two-electron Debye Hamiltonians. The reversal in the relative magnitudes of the singlet $v$. the triplet interparticle repulsions has been charted for the three models over a reasonably broad range of the pertinent parameters [15]. The overall behavior is in agreement with earlier results on the unscreened two-electron atom, showing interesting distinct features.

Singlet-triplet splitting was studied for two confined open-shell atomic systems as a function of the nuclear charge and of the radius of the confining sphere. In all the cases considered the total energy was confirmed to be lower in the triplet. The interelectronic repulsion was found to be lower in the singlet when the nuclear charge is low enough and the confining radius is large enough. The difference of interelectronic repulsions between the singlet and the triplet (denoted $\Delta C$ ) was found to be a decreasing function of the radius of the confining sphere, at a constant nuclear charge. However, as a function of the nuclear charge at a constant confining radius, this difference increases for large confining spheres, and exhibits a minimum at intermediate confining spheres. For tightly confining spheres $\Delta C$ decreases with increasing nuclear charge in the two-electron $1 s 2 p$ system, but increases with increasing nuclear charge in the four-electron $1 s^{2} 2 s 2 p$ system. This distinction can probably be understood as a consequence of the fact that the $1 s$ and the $2 p$ orbitals are very differently affected by the confining sphere, which is not the case for the $2 s$ and $2 p$ orbitals. While the contrast in the behavior of the difference between the singlet-triplet interelectronic repulsions in the free and in the confined atom follows from the analysis in [11], the transition between these two types of behavior is now clarified. 
In both systems studied $\frac{\partial E}{\partial R}<0$ (the energy decreases - increasing in absolute value - with increasing $R$ ). $\left|\frac{\partial E}{\partial R}\right|$ decreases with increasing $R$, vanishing at large $R$. More remarkably, it is higher in the singlet than in the triplet, due to the fact that the triplet wave function is more contracted, hence less affected by the confining sphere. Similarly, $\frac{\partial E}{\partial Z}<0$. Like the absolute value of the derivative with respect to $R,\left|\frac{\partial E}{\partial Z}\right|$ decreases with increasing $R$, remaining finite at $R \rightarrow \infty$ where it is equal to the expectation value of $\sum_{i=1}^{N} \frac{1}{r_{i}}, N$ being the number of electrons. As a function of $Z,\left|\frac{\partial E}{\partial Z}\right|$ increases. This is obvious, at least at large $R$, reflecting the contraction of the wave function upon increasing $Z$. The contraction of the triplet wave function relative to the singlet is also reflected in the fact that $\left|\frac{\partial E}{\partial Z}\right|$ is larger in the former than in the latter.

Investigation of the effects of milder confining potentials, such as a finite spherical square-well or a Gaussian potential, are worth-while extensions of the present study.

\section{References}

[1] Hund F., Zur deutung verwickelter spektren, insbesondere der elemente Scandium bis Nickel, Z. Phys., 1925, 33, P. 345-371.

[2] Slater J.C. The theory of complex spectra. Phys. Rev., 1929, 34, P. 1293-1322.

[3] Morgan III J.D., Kutzelnigg W. Hund's rules, the alternating rule, and symmetry holes. J. Phys. Chem., 1993, 97, P. $2425-2434$.

[4] Kutzelnigg W., Morgan III J.D. Hund's rules. Z. Phys. D, 1996, 36, P. 197-214.

[5] Davidson E.R. Single-configuration calculations on excited states of Helium. II. J. Chem. Phys., 1965, 42, P. 4199-4200.

[6] Koga T., Matsuyama H., J. Molina Molina, Dehesa J.S. Electron-pair densities of group 2 atoms in their ${ }^{1} \mathrm{P}$ and ${ }^{3} \mathrm{P}$ terms. Eur. Phys. J. D, 1999, 7, P. 17-23.

[7] Katriel J. A study of the interpretation of Hund's rule. Theoret. Chim. Acta, 1972, 23, P. 309-315. An interpretation of Hund's rule, ibid., 1972, 26, P. 163-170.

[8] Katriel J. and Pauncz R., Theoretical interpratation of Hund's rule. Adv. Quantum Chem., 1977, 10, P. 143-185.

[9] Katriel J. and Themelis S.I. Hund's rule in the doubly excited states of the Helium isoelectronic sequence. Int. J. Quantum Chem., 2012, 112, P. 2880-2893.

[10] Koga T. and Koshida Y. Roles of nuclear attraction and electron repulsion energies in the relative stability of atomic LS terms. $J$. Chem. Phys., 1999, 111, P. 53-54.

[11] Katriel J. and Montgomery Jr.H.E. Atomic vs. quantum dot open shell spectr. J. Chem. Phys., 2017, 146, P. 064104-(1-10).

[12] Sako T., Paldus J., Ichimura A. and Diercksen G.H.F. Origin of the first Hund rule and the structure of Fermi holes in two-dimensional He-like atoms and two-electron quantum dots. J. Phys. B, 2012, 45, P. 235001(1-13).

[13] Katriel J. and Montgomery Jr.H.E., Hund's rule in the two-electron quantum dot. Eur. Phys. J. B., 2012, 85, P. 394(1-4).

[14] Sarsa A., Buendía E., Gálvez F.J. and Katriel J. Singlet vs. triplet interelectronic repulsion in confined atoms. Chem. Phys. Letters, 2018, 702, P. $106-110$.

[15] Katriel J., Montgomery Jr.H.E. and Sen K.D. Hund's rule in the $(1 \mathrm{~s} 2 \mathrm{~s})^{1,3}$ S states of the two-electron Debye atom. Phys. Plasmas, 2018, 25, P. 092111(1-6).

[16] Pupyshev V.I. and Montgomery Jr.H.E. Spherically symmetric states of Hookium in a cavity, Phys. Scr., 2015, 90, P. 085401(1-8).

[17] Ugalde J.M., Sarasola C. and Lopez X. Atomic and molecular bound ground states of the Yukawa potential. Phys. Rev. A, 1997, 56, P. $1642-1645$.

[18] Mercero J.M., Fowler J.E., Sarasola C. and Ugalde J.M. Bound excited states of $\mathrm{H}^{-}$and $\mathrm{He}^{-}$in the statically screened Coulomb potential. Phys. Rev. A, 1998, 57, P. 2550-2555.

[19] Dai S.T., Solovyova A. and Winkler P. Calculations of properties of screened He-like systems using correlated wave functions. Phys. Rev. E, 2001, 64, P. 016408(1-9).

[20] Accad Y., Pekeris C.L. and Schiff B. S and P states of the Helium isoelectronic sequence up to Z = 10. Phys. Rev. A., 1971, 4, P. 516-536.

[21] Katriel J. The splitting of atomic orbitals with a common principal quantum number revisited: np vs. ns. J. Chem. Phys., 2012, 136, P. 144112(1-8).

[22] Knight R.E. and Scherr C.W. Two-electron atoms II. A perturbation study of some excited states. Rev. Mod. Phys., 1963, 35, P. $431-436$.

[23] Sanders F.C. and Scherr C.W. Perturbation study of some excited states of two-electron atoms. Phys. Rev., 1969, 181, P. 84-97.

[24] Blanchard P. Nonrelativistic-energy Z-expansion coefficients for singly excited S and P states of two-electron ions. Phys. Rev. A, 1976, 13, P. 1698-1701.

[25] Baker J.D., Freund D.E., Hill R.N. and Morgan III J.D. Radius of convergence and analytic behavior of the 1/Z expansion. Phys. Rev. A, 1990, 41, P. 1247-1273.

[26] Aashamar K., Lyslo G. and Midtdal J. Variational perturbation theory study of some excited states of two-electron atoms. J. Chem. Phys., 1970, 52, P. 3324-3336.

[27] Thakkar A.J. and Smith Jr.V.H. Compact and accurate integral-transform wave functions. II. The $2{ }^{1} \mathrm{~S}, 2^{3} \mathrm{~S}, 2{ }^{1} \mathrm{P}$, and $2^{3} \mathrm{P}$ states of the helium-like ions from He through $\mathrm{Mg}^{10+}$. Phys. Rev. A, 1977, 15, P. 16-22.

[28] Prudente F.V., Costa L.S. and Vianna J.D.M. A study of two-electron quantum dot spectrum using discrete variable representation method. J. Chem. Phys., 2005, 123, P. 224701(1-11).

[29] Lin Y.C., Fang T.K. and Ho Y.K. Quantum entanglement for helium atom in the Debye plasmas. Phys. Plasmas, 2015, 22, P. 032113(1-8). 